\title{
Эндокринотерапия рака молочной железы в период пре- и постменопаузы: соответствие клинической практики доказательной медицине
}

Получено 04.11.2019

Принято в печать 06.11.2019

DOI: $10.32471 /$ clinicaloncology.2663-466X.40.23202

\begin{abstract}
19-20 сентября 2019 г. в г. Винница состоялась международная научно-практическая конференция “Актуальные вопросы диагностики и лечения онкологических заболеваний репродуктивной сферы". Организаторами мероприятия выступили Национальный институт рака, Винницкий национальный медицинский университет им. Н.И. Пирогова, Подольский региональный центр онкологии, Общественная организация “Ассоциация онкологов Подолья", департамент здравоохранения Винницкой ОГА и Евро-Азиатская и ближневосточная Ассоциация заболеваний молочной железы (EURAMA). В ходе конференции были рассмотрены актуальные вопросы диагностики, лечения и профилактики различных онкологических заболеваний. В рамках секции маммологии был представлен доклад Николая Аникусько, кандидата медицинских наук, заведующего хирургическим отделением Киевского городского клинического онкологического центра, на тему “Эндокринотерапия рака молочной железы в пре- и постменопаузе. Соответствует ли клиническая практика доказательной медицине?".
\end{abstract}

\section{ИММУНОГИСТОХИМИЧЕСКОЕ ТИПИРОВАНИЕ ПРИ РМЖ И ПЕРСОНАЛИЗАЦИЯ ТЕРАПИИ}

1 января 2018 г. принята новая, 8-я версия ТNMклассификации Американского объединенного комитета по раку (American Joint Committee on Cancer - AJCC), которая, к сожалению, еще не везде адаптирована в украинских лечебных учреждениях онкологического профиля, поскольку не все из них имеют возможность широко использовать в своей работе иммуногистохимические методы типирования. Тем не менее на сегодня иного пути развития в этом направлении нет, поскольку без использования иммуногистохимического типирования диагностировать и эффективно лечить онкологических больных практически невозможно.

Почему эта тема очень актуальна в отношении рака молочной железы (РМЖ)? 75\% всех типов РМЖ - это гормонзависимые опухоли, что определяется иммуногистохимическими методами по наличию соответствующих рецепторов. Известно, что гормональная чувствительность связана с экспрессией рецепторов эстрогена и/или прогестерона на поверхности опухолевых клеток. При этом в последние годы в данном направлении произошли серьезные эволюционные изменения. Так, если в 1990-е годы гормонположительные опухоли определялись при наличии более $15 \%$ опухолевых клеток, экспрессирующих рецепторы, то уже в 2000-е годы этот показатель составлял более $10 \%$, в 2010-е годы - более 5\%. И наконец в 2015 г. Американская ассоциация онкологов определила, что при наличии более $1 \%$ опухолевых клеток, экспрессирующих рецепторы эстрогена и/или прогестерона, опухоль является гормончувствительной, и, соответственно, в этом случае показано назначение эндокринотерапии. Эти изменения связаны с тем, что во многих странах иммуногистохимическое типирование опухолей улучшилось за счет стандартизации подходов, использования современных маркеров и т.д.

Гормончувствительные опухоли являются очень индолентными, они не обладают значительным агрессивным потенциалом и имеют прогнозируемое течение. Как правило, гормонзависимые опухоли наиболее часто отмечают в возрасте старшее 55 лет. Врачи в этом случае располагают достаточно неплохими химиотерапевтическим и лекарственным арсеналом. При этом терапия относительно доступна и обладает низкой токсичностью, обеспечивая высокий уровень 5-летней выживаемости.

Докладчик привел результаты клинического исследования, в ходе которого проводили сравнение химиотерапии

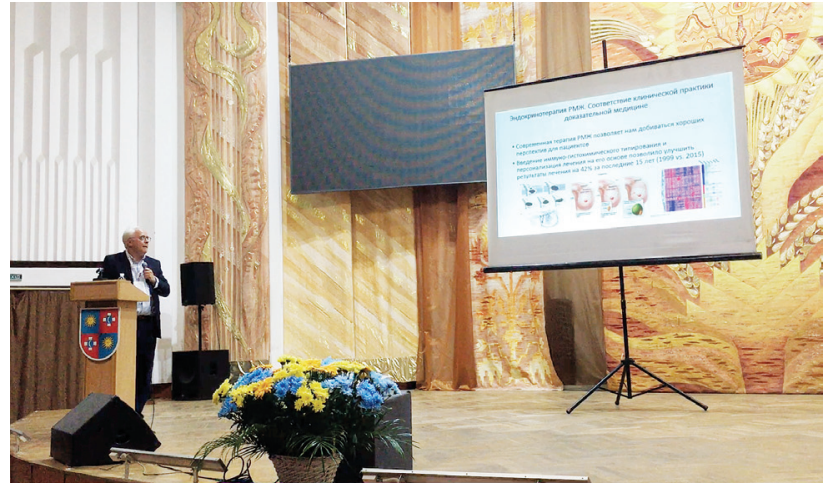

и гормонотерапии в неоадъювантном режиме у пациентов в период постменопаузы с гормончувствительным РМЖ. Согласно результатам исследования было показано, что клинический ответ после гормонотерапии был выше по сравнению с химиотерапией у пациентов с гормончувствительными опухолями и отмечалась в 1,5 раза более высокая степень выполнения органосохраняющих операций. В то же время достижения полного патоморфологического ответа (pathological complete response - pCR) у пациентов с люминальными типами РМЖ в большинстве случаев не происходило.

Гормонположительные опухоли подразделяются на люминальный тип А и люминальный тип В. В свою очередь, люминальный рак типа В может быть высокопролиферативным или трижды позитивным. РМЖ люминального типа А хуже реагирует на применение химиотерапевтических препаратов, нежели на гормонотерапию, демонстрируя в последнем случае снижение индекса пролиферативной активности Ki 67. Но при этом редко удается достичь полного pCR. В свою очередь, высокопролиферативный РМЖ также отвечает на гормонотерапию (отмечается снижение Ki 67), но может демонстрировать определенную выгоду от применения химиотерапии. При этом по сравнению с люминальным раком тип A на фоне лечения чаще достигается pCR. Трижды позитивный РМЖ (гормонположительный, HER2+) - это биологический подтип, который хуже отвечает на гормонотерапию и реагирует на химиотерапию. При этом проблемой является низкий уровень достижения $\mathrm{pCR}$ при неоадъювантной анти-HER2+-химиотерапии. Поэтому при трижды позитивном РМЖ показана двойная анти-HER2+-терапия. Важно, что пересечение между сигнальными путями HER2 и рецепторов эстрогена может играть существенную роль в развитии рефрактерности опухоли к лечению. 


\section{РАСШИРЕННАЯ ЭНДОКРИНОТЕРАПИЯ И РИСК РЕЦИДИВА}

Эндокринотерапия РМЖ как подход системного лечения гормонположительного РМЖ включает адъювантную, неоадъювантную и паллиативную гормонотерапию. При этом доказательная медицина дает возможность использовать эндокринотерапию РМЖ в объеме, необходимом для достижения поставленной цели, и менять ее при необходимости.

Основной целью применения эндокринотерапии при гормонположительном РМЖ сегодня является оптимизация выживаемости пациентов при минимальной токсичности лечения. Н. Аникусько выделил 3 пути возможного повышения выживаемости больных РМЖ, а именно: улучшение лечения пациентов, находящихся в группе повышенного риска развития рецидива, обеспечение доступа к высококачественной помощи для большинства больных, получающих субоптимальное лечение, и минимизация терапии у пациентов, нуждающихся в деэскалации лечения. С какими вызовами сталкиваются врачи-онкологи при лечении пациентов с гормончувствительными опухолями? Сегодня актуальным является вопрос повышения уровня 10-летней выживаемости, которая сокращается из-за повторного рецидивирования заболевания и метастазирования. Очень важно, что после 5 лет гормонотерапии часто развивается рефрактерность к лечению, часто обусловленная ESR1-мутацией. При этом разработаны схемы последовательности гормонотерапии и существует ряд препаратов, улучшающих ответ на гормонотерапию, что позволяет усилить и продлить гормонотерапию.

Анализ результатов лечения пациентов с метастатическим гормонположительным РМЖ показал, что у $63 \%$ больных прогрессирование патологии отмечали через 7 и более лет после операции или через 3-4 года после окончания 5-летней гормональной терапии, еще у $14 \%$ пациентов отмечалось прогрессирование заболевания через 4-7 лет после операции на фоне нарушения режима гормональной терапии или самостоятельного преждевременного ее прекращения. Кроме того, было показано, что именно индолентность гормончувствительных опухолей сохраняет латентный риск развития рецидива еще в течение 20 лет после окончания лечения.

Таким образом, клиницистам необходимо широко применять в своей работе инструменты прогноза, которые являются ключевыми факторами при выборе гормонотерапии. Н. Аникусько подчеркнул важность использования надежных подходов, позволяющих предвидеть резидуальный риск рецидива. Сегодня существует большое количество различных биомаркеров, в том числе и геномных, которые влияют на прогноз течения заболевания. Так, при оценке риска развития рецидива РМЖ учитываются клинико-патологические факторы, молекулярный профиль опухоли, экспрессия белков (протеомика), различие в ответе на гормонотерапию, в том числе наличие положительного влияния расширенной гормонотерапии. Биомаркеры должны обладать определенными свойствами: аналитическая достоверность (воспроизводимость и точность), клиническая достоверность и клиническая польза. Выделяют 4 группы наиболее часто использующихся клинических биомаркеров: анатомические (TNM), иммуногистохимические (люминальный тип А, В и т.д.), генетические (BRCA, PALB, TP53 и т.Д.) и геномные. Для определения риска развития рецидива при РМЖ разработан ряд методик прогноза течения заболевания, например OncotypeDX, PEPI, CTS5 Score и т.д.

Длительное время одним из наиболее популярных был Ноттингемский прогностический индекс (NPI), в последнее время все большее значение приобретают предоперационный эндокринный прогностический индекс (preoperative endocrine prognostic index - PEPI), а также индекс пролиферативной активности Кі 67.

Ki 67 - фактор прогноза, изменение которого указывает на необходимость применения химиотерапии у пациентов с люминальным РМЖ типа А. Кі 67 помогает идентифицировать пациентов, не нуждающихся в химиотерапии, а также получить представление о биологической основе эффективности гормональных препаратов. Кі 67 позволяет оценить биомаркеры ответа и механизмы устойчивости при остаточном раке после лечения, а также используется при разработке механизмов лечения заболеваний, устойчивых к эндокринной терапии. В частности, в исследованиях IMРАСТ и АТАС было показано, что анастрозол демонстрирует преимущество перед тамоксифеном относительно супрессии Кі 67 и выживаемости без прогрессирования. Таким образом, оценка Ki 67 после 2-недельного приема анастрозола позволяет прогнозировать вероятность рецидивирования у пациентов с РМЖ.

В другом исследовании РОЕТІС пациенты с гормонзависимым инвазивным РМЖ были рандомизированы на две группы: первая получала летрозол за 2 нед до операции, вторая была прооперирована без лечения. Если Кi 67 в биоптате был 15 и менее, то 5-летний риск рецидива составлял 4,5\%, а если уровень Кі 67 оставался высоким после 2-недельного приема летрозола, то 5-летний риск рецидива соответствовал $19,6 \%$.

В свою очередь, прогностический алгоритм PЕРI учитывает данные о размере опухоли, уровне вовлеченности лимфоузлов, уровне Ki 67 и гормончувствительности опухоли. Таким образом, PEPI является эквивалентом рCR и позволяет на основе полученных с его помощью данных проводить де-/эскалацию системной терапии.

Еще одним фактором, который, согласно исследованиям, может предопределять развитие рецидива, является наличие циркулирующих опухолевых клеток. При этом чем больше выявляется таких клеток, тем выше вероятность рецидива. Так, при наличии 1 циркулирующей опухолевой клетки на 7,5 мл крови риск рецидива оценивается на уровне $16,7 \%$, а если таких клеток более 2 , риск существенно повышается до $83,3 \%$. В целом наличие циркулирующих опухолевых клеток повышает вероятность рецидива в 13 раз.

В 2018 г. на симпозиуме «San Antonio Breast Cancer Symposium» был представлен доклад, посвященный оценке 20-летнего риска рецидива РМЖ после прекращения 5-летней эндокринотерапии, в котором были проанализированы результаты 88 клинических исследований, в которых приняли участие почти 63 тыс. пациентов с диагностированным РМЖ в 1976-2011 гг. Согласно полученным результатам, размер опухоли, степень дифференцирования, количество вовлеченных лимфатических узлов являются предикторами развития рецидива. Основываясь на данных результатах, был предложен индекс для определения риска развития рецидива РМЖ после 5-летней гормонотерапии - CTS5 Score, соответствующий калькулятор доступен онлайн или в виде приложения. Данный индекс пришел на смену Ноттингемскому прогностическому индексу и широко используется, он учитывает размер опухоли, степень дифференцировки, количество вовлеченных лимфатических узлов и возраст пациента.

В 2016 г. Нобелевская премия в области физиологии и медицины была присуждена Есинори Осуми за открытие механизмов автофагии. Это открытие заложило основы для развития ряда директивных направлений, в том числе повлияло на взгляды относительно механизмов развития и прогрессирования злокачественных новообразований. Так, считается, что механизмы рецидивирования при онкопатологии, особенно это характерно для люминальных типов РМЖ, связаны со способностью нормальных клеток костной ткани остеокластов - поглощать и длительное время удерживать, не переваривая, стволовые опухолевые клетки, таким образом защищая их от воздействия внешней среды и лекарственных агентов. Таким образом, опухолевые клетки длительно консолидируются внутри костей. Это открытие объяснило высокую эффективность остеокласт-активных агентов в отношении риска развития рецидива при онкопатологии. 
На основании каких факторов следует принимать решение о проведении расширенной гормонотерапии? В руководстве ASCO по использованию биомаркеров для определения выбора адъювантной системной терапии при раннем инвазивном РМЖ отмечается, что клиницисты не должны использовать многофакторные генные экспрессии или исследование белков при принятии решения о расширенной эндокринной терапии. В то же время Н. Аникусько привел данные о нескольких проспективных рандомизированных исследованиях, в которых оценивалась возможность применения генетических маркеров для принятия решения о проведении расширенной гормональной терапии при РМЖ. В частности, это обсервационное когортное 5-летнее клиническое исследование TAILORx, в котором принимали участие пациенты с РМЖ без вовлечения лимфатических узлов; рандомизированное когортное клиническое исследование RxPonder, в котором принимали участие пациенты с РМЖ с вовлечением 1-3 лимфатических узлов; и обсервационное рандомизированное 5-летнее клиническое исследование MINDACT, в котором принимали участие пациенты с РМЖ без и с вовлечением 1-3 лимфатических узлов.

Н. Аникусько более детально остановился на исследовании TAILORx, результаты III фазы которого были представлены в 2019 г. на ASCO. В частности, сравнивали результаты химиоэндокринной терапии и эндокринной терапии у пациентов с гормончувствительным HER2-PМЖ без вовлечения лимфоузлов со средними показателями вероятности рецидивирования согласно 21-генной панели. Как известно, выделяют несколько категорий пациентов в зависимости от риска рецидивирования. У пациентов с низким риском рецидивирования не отмечают положительного влияния химиотерапии, они отвечают на гормонотерапию. Пациенты с высоким риском рецидивирования отвечают на комбинацию химио- и гормонотерапии. Но существует определенная категория пациентов (со средним риском рецидивирования), чей удельный вес достаточно велик, данные относительно эффективности различных видов терапии для которых противоречивы. Решить именно эту задачу было призвано исследование TAILORX, в котором приняли участие пациенты в возрасте $18-75$ лет с риском рецидива 11-25 согласно Recurrence Score (RS). Они были рандомизированы на две группы: одна получала только эндокринную терапию, вторая - эндокринную и химиотерапию. Согласно полученным результатам было показано, что показатели 9-летней безрецидивной и 9-летней общей выживаемости в обеих группах пациентов статистически не отличались. В ходе анализа пациенты были разделены на подгруппы в зависимости от уровня риска развития рецидива: $11-15,16-20$ и $21-25$ согласно RS. В рамках этих подгрупп было отмечено различие относительно риска отдаленного метастазирования 1,6 и 6,5\% в пользу комбинированной схемы лечения для подгруппы 16-20 RS и 21-25 RS соответственно, однако эти различия не были статистически достоверными. Итак, добавление к схеме лечения пациентов со средним уровнем риска рецидивирования (11-25 RS) химиотерапии не дает преимуществ по сравнению с одной только гормонотерапией. Таким образом, химиотерапия не дает преимуществ при применении у пациентов с уровнем риска рецидивирования 0-25 RS, на долю которых приходится примерно 85\% пациентов с гормончувствительным HER2-PМЖ без вовлечения лимфоузлов, и показана только пациентам с высоким риском рецидивирования 26-100 RS. Можно ли экстраполировать эти данные на пациентов с гормончувствительным РМЖ с вовлечением лимфоузлов? Безусловно, необходимы дополнительные исследования.

В свете вышеприведенных данных актуальным становится вопрос раннего определения рецидива как следующий шаг после прогнозирования его потенциального развития. При этом раннее определение метастатического заболевания позволяет выявить пациентов, которых можно вылечить на раннем этапе прогрессирования. В свою очередь, лечение неопределяемого микрометастатического заболевания с помощью адъювантной терапии позволит снизить риск рецидива и смерти пациента. Это не отменяет важность выявления номинальной опухоли, которая, как правило, характеризуется низким уровнем накопленных онкогенных мутаций, и инициации терапии на ранних этапах.

Таким образом, полученные фундаментальные научные данные позволяют обосновать применение таких терапевтических стратегий при гормонположительном РМЖ, как расширенная гормонотерапия, HER2-агенты и остеокласт-таргетные препараты. В этом контексте Н. Аникусько подробно остановился на возможных стратегиях по уменьшению количества отсроченных рецидивов. В первую очередь это применение в адъювантном режиме тех препаратов, которые подтвердили свою эффективность при метастатическом процессе: селективные антагонисты эстрогеновых рецепторов - фулвестрант (Фазлодекс), ингибиторы CDK 4/6, mTOR-ингибиторы и PARP-ингибиторы. В настоящее время активно проводятся клинические исследования эффективности и профиля безопасности данных препаратов, и их результаты ожидаются в ближайшем будущем. Также перспективными являются новые направления терапии, такие как ингибиторы HDAC, ингибиторы PI3 киназ и др.

В свою очередь, продолжение гормонотерапии после 5 лет (расширенная гормонотерапия) дает очень хорошие результаты. Так, уровень абсолютной выгоды относительно снижения смертности в этом случае составляет $1-4 \%$. В частности, длительное применение тамоксифена (более 5 лет) снижает риск рецидивирования на 1/3, применение ингибиторов ароматазы после тамоксифена - дает относительное снижение риска на $1 \frac{1}{2}$. Аналогичные данные для блокаторов ароматазы после применения этой же группы препаратов оказались противоречивыми.

В другом метаанализе, проведенном Early Breast Cancer Trialists' Collaboration Group, оценивалась эффективность продолжения лечения ингибиторами ароматазы после 5 и более лет эндокринотерапии. Метаанализ объединил данные 11 рандомизированных клинических исследований, в которых приняли участие более 22 тыс. женщин, получавших гормонотерапию при РМЖ (5 лет применялся тамоксифен; 5-10 лет применялся тамоксифен, потом ингибиторы ароматазы; 5 лет применялись ингибиторы ароматазы). Наибольшая степень снижения риска возникновения отдаленных рецидивов и смертности отмечалась в группе, в которой происходила замена тамоксифена на ингибиторы ароматазы. При этом в рамках 10-летнего наблюдения было отмечено снижение на 2,5\% риска всех рецидивов в целом, риска отдаленного метастазирования - на $1,1 \%$ и риска смерти, связанной с РМЖ, - на $0,3 \%$. При этом существенно снижалось количество контралатеральных и изолированных локальных рецидивов, а также отдаленных метастазов. Кроме того, было показано, что расширенная гормональная терапия демонстрирует лучшие результаты при применении у пациентов с большим количеством пораженных лимфатических узлов. Так, применение гормональной терапии (ингибиторы ароматазы) у пациентов без пораженных лимфоузлов позволило снизить риск развития рецидива на $1,1 \%$, аналогичный показатель для пациентов с 4 и более пораженными лимфоузлами составил $7,7 \%$.

Таким образом, применение ингибиторов ароматазы после 5 и более лет гормонотерапии позволяет достичь снижения рецидивирования у женшин, получавших в течение 5 лет тамоксифен, примерно на $35 \%$ и снижения риска рецидивирования у женщин, получавших ингибиторы ароматазы (с или без тамоксифена), примерно на 20\%. При этом наиболее выраженное снижение риска рецидивирования при расширенной терапии ингибиторами ароматазы отмечено в первые 2 года после окончания терапии тамоксифеном, но не раньше 
чем на 3-й год лечения ингибиторами ароматазы. Также необходимо подчеркнуть более существенный положительный эффект от применения ингибиторов ароматазы у пациентов с вовлечением лимфоузлов. Среди негативных аспектов расширенной гормонотерапии необходимо отметить повышение риска переломов на $25 \%$.

Подводя промежуточные итоги, докладчик отметил, что накопленные результаты рандомизированных исследований позволяют проводить коррекцию лечения на основании ответа опухоли на химиотерапию. Широкое применение неоадъювантной полихимиотерапии у пациентов с ранним РМЖ привело к тому, что хирурги и радиологи часто отмечают pCR. Это приводит к возникновению множества вопросов о том, каким должно быть дальнейшее оптимальное лечение для снижения риска рецидива при минимальной токсичности. Недостаточный уровень ответа опухоли на проводимое лечение дает онкологам второй шанс воздействовать на нее.

10-летняя гормонотерапия показала, что при длительном применении ингибиторов ароматазы может развиваться устойчивость к лечению. Первичная опухоль обладает генетической гетерогенностью, и при назначении определенных препаратов происходит селекция опухолевых клеток с мутациями, ответственными за устойчивость к проводимой терапии, в $18 \%$ случаев при метастатическом РМЖ это мутация ESR1. При этом развитие устойчивости при приеме ингибиторов ароматазы отмечается примерно у $50 \%$ пациентов. Определение ESR1-мутации с помощью полимеразной цепной реакции в $56 \%$ случаев позволяет выявить риск генетического рецидива за 7 мес до его клинического проявления.

В одном из исследований - SoFEA, в котором еще применялись низкие дозы фулвестранта (250 мг), было показано, что основным путем преодоления ESR1-мутации является применение фулвестранта. Он показал более высокую эффективность по сравнению с экземестаном.

Сегодня в ряде исследований было показано преимущество фулвестранта по сравнению с ингибиторами ароматазы в качестве первой линии терапии у пациентов с гормончувствительным местно-распространенным/метастатическим PMЖ. Так, FIRST - рандомизированное открытое многоцентровое исследование II фазы, в котором изучалась эффективность и профиль безопасности фулвестранта в дозе 500 мг по сравнению с анастрозолом 1 мг в качестве первой линии терапии у женщин в период постменопаузы с гормончувствительным местно-распространенным/метастатическим РМЖ. Согласно полученным результатам, при применении фулвестранта выживаемость без прогрессии увеличивалась более чем на 10 мес по сравнению с применением анастрозола. Так, медиана времени до прогрессирования составила 23,4 мес для фулвестранта против 13,1 мес для анастрозола, а снижение риска прогрессирования составило $34 \%$. Кроме того, в исследовании FIRST было показано улучшение общей выживаемости при лечении фулвестрантом в дозе 500 мг по сравнению с анастрозолом в качестве первой линии терапии при эстрогенрецептор положительном (ER+) распространенном РМЖ, со снижением риска смерти приблизительно на $30 \%$. Так, было продемонстрировано, что эндокринная монотерапия фулвестрантом в дозе 500 мг обладает более высокой эффективностью по сравнению с ингибиторами ароматазы третьего поколения.

В другом исследовании была изучена эффективность фулвестранта 500 мг в качестве первой линии терапии по сравнению с анастрозолом. Исследование FALCON - рандомизированное двойное слепое клиническое исследование III фазы, в котором приняли участие женщины в период постменопаузы с гормончувствительным местно-распространенным или метастатическим РМЖ, которые ранее не получали эндокринную терапию. Согласно полученным результатам, применение фулвестранта ассоциировалось со статистически значимым улучшением выживаемости без прогрессирования по сравнению с анастрозолом. Так, медиана выживаемости без прогрессирования составила 16,6 мес при применении фулвестранта и 13,8 мес - в группе, получавшей анастрозол (разница 2,8 мес). При этом необходимо подчеркнуть, что фулвестрант высокоэффективен у пациентов без висцеральных метастазов. Так, медиана выживаемости без прогрессирования в подгруппе пациентов без висцеральных метастазов составила 22,3 мес при применении фулвестранта и 13,8 мес - в группе, получавшей анастрозол (разница 8,5 мес). Таким образом, благодаря результатам исследования FALCON, анастрозол утрачивает свои лидирующие позиции, а фулвестрант является более клинически эффективным препаратом первой линии терапии метастатического РМЖ.

Таким образом, гормональная терапия является высокоэффективным методом системной терапии гормончувствительного РМЖ. При этом в адъювантном режиме ингибиторы ароматазы продемонстрировали преимущества по сравнению с тамоксифеном. В то же время результаты исследований свидетельствуют в пользу 10 -летней гормональной терапии по сравнению с 5-летней, особенно в случае вовлечения $1-3$ лимфоузлов и при замене тамоксифена на ингибиторы ароматазы. В то же время длительный прием ингибиторов ароматазы может приводить к развитию рефрактерности за счет селекции опухолевых клеток с ESR1-мутацией. Фулвестрант является первым выбором для старта эндокринотерапии у пациентов с гормонположительным распространенным РМЖ, который показал свое клиническое преимущество по сравнению с ингибиторами ароматазы третьего поколения.

Евгения Гончарук 